\title{
Effects of Different Methods for Achieving Situational Awareness on Cerebral Blood Flow in Nurses in an Observational Setting
}

\author{
Ayako Nishimura \\ Tokyo Medical University, Faculty of Medicine, School of Nursing, Tokyo Medical and Dental University, Graduate School of Health Care Sciences, Tokyo, Japan \\ "Corresponding author: Ayako Nishimura, Tokyo Medical University Faculty of Medicine, School of Nursing,6-1-1 Shinjuku, Shinjuku-ku, Tokyo 160-8402, Japan, Tel: \\ +81-(0)3-3351-6141 ext. (778); Fax: +81-(0)3-3351-3691; E-mail: nishiaya@tokyo-med.ac.jp \\ Received date: October 20, 2016; Accepted date: November 21, 2016; Published date: November 28, 2016 \\ Copyright: @ 2016 Nishimura A . This is an open-access article distributed under the terms of the Creative Commons Attribution License, which permits unrestricted use, \\ distribution, and reproduction in any medium, provided the original author and source are credited.
}

\begin{abstract}
When nurses make natural decisions in practical settings, appropriate actions to decrease errors and improve patient safety follow decisions based on proper situational awareness that includes integration of past experiences, the current situation, and future predictions. We established an observational setting where nurses performed environmental arrangement to serve as a setting where they could practice routine situational awareness. This provided an opportunity to consciously practice situational awareness during observation in this setting and then measure cerebral blood flow in the same setting. The aims of this design were to elucidate the effects on cerebral blood flow by comparing different methods of practicing situational awareness and to investigate effective methods of practicing situational awareness.
\end{abstract}

We performed a randomized, comparative study of different situational awareness methods with 61 nurses with experience of working in hospital wards. These nurses were randomly assigned to one of three groups according to the different situational awareness methods (writing, oral, and implicit groups). We measured cerebral blood flow changes using the near infrared spectroscopy device placed at two positions on the participant's forehead. A visual analog scale was used for the subjective evaluation of participant characteristics. For statistical analyses, we used a Wilcoxon test to compare the results for each group before and after implementing situation awareness methods; Kruskal-Wallis or Mann-Whitney tests and Bonferroni procedure were used to compare the results between three groups.

The provision of opportunities to practice situational awareness had the following effects on cerebral blood flow: (1) left and right total $\mathrm{Hb}$ values increased after situational awareness in both the writing and oral groups; (2) right total $\mathrm{Hb}$ values in the pre-situational awareness task in the oral group were high; and (3) left and right total $\mathrm{Hb}$ values during situational awareness were high in the oral group.

The use of writing and oral methods by nurses for situational awareness was found to promote the brain activity not only during the process of situational awareness but also during the observations after situational awareness.

Keywords: Nurse, Situation awareness, Observation, Near infrared spectroscopy

\section{Background}

Healthcare professionals make various natural decisions based on their experiences and knowledge to decrease errors and improve patient safety [1]. Natural decision making [2] consists of three steps, i.e., situational awareness, decision, and performance of action, and the outcomes of the action are fed back. Therefore, even highly skilled experts fail to make appropriate natural decisions if the situation is perceived incorrectly. When nurses make natural decisions in practical settings, decisions that are based on proper situational awareness, including the integration of past experiences, the current situation, and future predictions, lead to appropriate actions [3]. Therefore, situational awareness cannot be achieved unconsciously by the individual on the basis of previous individual knowledge or experience. It is also not dependent on the individual's abilities or responsibilities. Instead, it is important to encourage appropriate behavior by creating opportunities to promote appropriate situational awareness. There have been previous reports that have measured situational awareness using an assessment tool [4] and that have discussed the importance of integrating information [1] and the necessity for education and training [5]. However, the effects of creating opportunities for situational awareness and methods to promote situational awareness have not been clarified.

The Ministry of Education, Culture, Sports, Science and Technology initiated the "Brain Science and Education" project in Japan in 2001. This project aims to investigate topics such as attentiveness, motivation, curricula, and teaching methods as well as to provide an objective evaluation and determine a scientific rationale. Given these current conditions, the use of near infrared spectroscopy (NIRS) to evaluate brain activity has been attracting attention on a global scale in recent years [6,7]. NIRS can estimate changes in blood flow in the cerebral cortex through probes on the surface of the skin, and can monitor these changes over time in a non-invasive manner, even during tasks that require the subject to move [8]. Therefore, NIRS is being increasingly used in studies related to teaching and learning. Neurovascular coupling refers to the changes in cerebral hemodynamics that occur when cerebral blood vessels dilate during 
brain activity. This mechanism is used as an index to evaluate cerebral activity, [9] and studies have shown that cerebral blood flow increases during intense thinking and ceases to increase when understanding is achieved $[10,11]$. In conclusion, if cerebral blood flow increases during situational awareness, it signifies that the person is thinking intensely about situational awareness. Accordingly, we believed that this might affect subsequent actions.

Based on this information, we considered it useful to offer nurses opportunities to consciously practice situational awareness to promote situational awareness. We also considered that evaluating cerebral activity while using different situational awareness methods would provide an insight into methods to effectively practice situational awareness.

\section{Aim}

We established an observational setting where nurses performed environmental arrangement to serve as a setting where they could practice routine situational awareness. This provided an opportunity to consciously practice situational awareness during observation in this setting and then measure cerebral blood flow in the same setting. The aims of this design were to elucidate the effects on cerebral blood flow by comparing different methods of practicing situational awareness and to investigate effective methods of practicing situational awareness.

\section{Methods}

\section{Research Design}

We performed a randomized, comparative study of different situational awareness methods.

\section{Participants}

Subjects were required to be experienced in environmental arrangement as the observational setting that was established posed the challenge of environmental arrangement to nurses. Therefore, we selected nurses with current or previous experience of working in a hospital ward. If nurses were no longer active in wards, they could still be included if they had performed ward work within the preceding 3 years. The experiment was performed between January 9 and March 3, 2014.

Participants were recruited through the University home page. A total of 62 nurses in the Kanto region (one metropolitan area and four prefectures) in Japan volunteered to participate. One applicant was excluded as the applicant did not meet the inclusion criteria. The study population therefore included 61 participants. Written informed consent was obtained from all subjects before their enrollment in the study.

The participants were randomly assigned to one of the three groups based on the different situational awareness methods using a random number table (writing group [ $\mathrm{n}=21]$, oral group [ $\mathrm{n}=20]$, and implicit group $[\mathrm{n}=20])$.

\section{Protocol}

A private room was used as a laboratory. The humidity and temperature were adjusted to $19.4 \pm 2.3^{\circ} \mathrm{C}$ and $28.7 \pm 4.3 \%$, respectively, to avoid affecting implementation of the task (Figure 1).

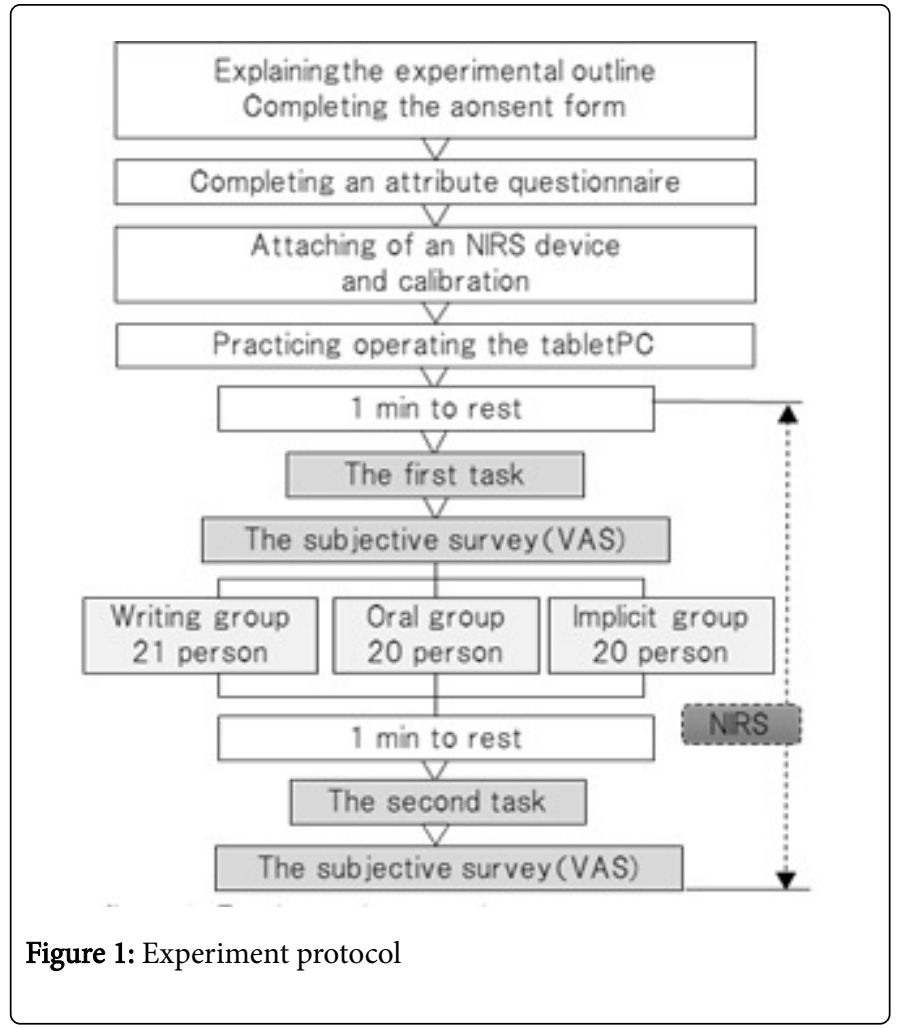

Researchers explained the experiment to participants and then asked them to answer a self-administered questionnaire. Participants were asked to sit on a chair in front of a tablet PC (Windows 8 DELL Optiplex 9010AIO) placed on a desk. An NIRS device was attached to the center of the forehead of each participant during the study and then was performed.

A description of the task was displayed on the tablet PC, and researchers explained to participants that operation and selection could be performed by touching the screen during the task. All participants were given a 5 -min period to practice the tablet PC operations and then given $1 \mathrm{~min}$ to rest. The following instructions were provided: (1) The participant would observe a hospital room (to be displayed as a still image on the PC screen) as he/she would do in actual environmental arrangement and (2) The participant would be then required to touch items pertinent to environmental arrangement on the screen. During the task, a still image was displayed on the screen that showed a living environment in a hospital room, and participants were asked to observe the hospital room image as nurses.

After the first task, participants filled out a subjective survey (using a visual analog scale [VAS]) and were given an opportunity to practice situational awareness. Participants in the writing, oral, and implicit groups performed the tasks using methods that were assigned to each group. They were then given another a 1-min break, performed the second task, completed the subjective survey (VAS), and concluded the experiment.

To prevent information from being leaked, the experiment was conducted on one participant at a time. Participants were instructed to refrain from disclosing the contents of the experiment. A single researcher conducted the entire experiment in a reproducible manner to increase the reliability of the experiment. 


\section{Intervention method}

The opportunities provided for practicing situational awareness involved participants answering questions while looking at the still image which represented the task. Questions about situational awareness were as follows: (1) perception of elements in the image (current situation); (2) comprehension of current situation, and (3) projection of future status. These three components are based on Endsley's internal processes of situational awareness [2]. Participants had $60 \mathrm{~s}$ to answer each question. Participants in the writing group were requested to enter the above mentioned contents into the tablet PC, while those in the oral group answered questions verbally to the tablet PC. Those in the implicit group were requested to remember what they thought.

\section{Evaluation methods}

\section{Attributes}

Information on participant's age, sex, educational background, and experience in the ward was collected using a self-administered questionnaire. Participant characteristics, including intuitive judgment, logical judgment, tablet PC usage, fatigue felt during the experiment, and drowsiness felt during the experiment, were also evaluated using a VAS.

\section{Cerebral blood flow}

As the prefrontal cortex is involved in short-term retention of information, processing of working memory, and an executive function necessary to perform a series of purposeful activities [12,13], we measured cerebral blood flow changes at two positions on the participant's forehead using the NIRS device (HOT121B: HITACHI). The $1 \mathrm{ch}$ and $2 \mathrm{ch}$ probes were placed over the right and left prefrontal cortex regions, respectively, with the Fz point (frontal midline) as the reference, in accordance with the international 10-20 system. We measured the Brodmann area 10, which is a part of the frontal association area that controls higher mental functions and natural decisions, as an index. The measurements were performed continuously over time from the time of calibration to the completion of the second task.

NIRS is a method that involves illumination of the scalp with near infrared light of different wavelengths and measuring changes in cerebral blood flow based on differences in absorbance. The method uses total $\mathrm{Hb}$ as an index of blood flow. Because data from NIRS measurements indicate relative changes in total $\mathrm{Hb}$ in reference to the cerebral blood flow at calibration, the possible range of total $\mathrm{Hb}$ values varies among different individuals. We thus focused on the maximum amplitude value during the task and used normalized total $\mathrm{Hb}$ values ( $\mathrm{z}$ score) for the analyses. In this study, after subjecting total $\mathrm{Hb}$ obtained with NIRS to 10 -s moving average processing, $\mathrm{z}$ score $=(\mathrm{xi}-$ $\mathrm{X}) / \mathrm{SD}$, the maximum amplitude value (xi) divided by the standard deviation $(\mathrm{SD})$ for the mean total $\mathrm{Hb}(\mathrm{X})$ in the 10 -s rest period, immediately before starting the task, was calculated.

\section{VAS}

When performing subjective evaluation during the tasks, a VAS was used to evaluate whether participants "consciously observed," "repeatedly observed," or "observed while thinking about a reason". A score of 100 was assigned when participant's observation of an item met the above criteria very well, whereas a score of 0 was assigned when the observation did not meet the criteria at all.

\section{Statistical analysis}

For statistical analyses, we used a Wilcoxon test to compare the results for each group before and after implementing situation awareness methods; Kruskal-Wallis or Mann-Whitney tests and Bonferroni procedure were used to compare the results between three groups. Before these tests, the Shapiro-Wilk test was used to confirm that all the data did not fall under a normal distribution curve. All statistical analyses were performed using IBM SPSS Statistics 24, and the level of significance was set at $5 \%$.

\section{Ethics}

The study was performed with the approval of the Tokyo Medical and Dental University Ethical Review Board. Before the experiment, we provided the participants with verbal and written explanations regarding the study purpose, before obtaining informed consent. The participants were informed that they would be placed at no disadvantage whether or not they participated and that their privacy would be protected. In addition, they were informed that the information obtained would not be used for any purpose other than the study, their personal identity would be protected and not specified in publications, and they could withdraw from participation during the experiment.

\section{Results}

\section{Participant attributes}

No significant differences were found in participant age, sex, educational background, years of experience, and ward work experience, which minimized the scope for bias as a group (Table 1). There were no differences in judging, mental summarization, verbal description, writing, touch panel usage, drowsiness on the day of experiment, and fatigue on the day of experiment (Table 2). These results allowed us to conclude that there was no bias among the participants, and all participants were equivalent except for the intervention factor.

\begin{tabular}{|c|c|c|c|c|c|c|}
\hline & & All & Writing & Oral & Implicit & \\
\hline & & $n=16$ & $n=21$ & $\mathrm{n}=\mathbf{2 0}$ & $\mathrm{n}=\mathbf{2 0}$ & $\begin{array}{l}p \\
\text { value }\end{array}$ \\
\hline Age & $\begin{array}{l}\text { Median } \\
\text { (Quartile } \\
\text { deviation } \\
\text { ) }\end{array}$ & $\begin{array}{l}29.0(Q 3.0 \\
)\end{array}$ & $\begin{array}{l}29.0(Q 2.5 \\
)\end{array}$ & $\begin{array}{l}28.5(\mathrm{Q} 2.0 \\
)\end{array}$ & $\begin{array}{l}28.0(Q 3.5 \\
)\end{array}$ & 0.724 \\
\hline \multicolumn{7}{|l|}{ Sex } \\
\hline Male & & $4(6.5 \%)$ & $1(1.6 \%)$ & $1(1.6 \%)$ & $2(3.2 \%)$ & \\
\hline Female & & $\begin{array}{l}57(93.5 \% \\
\end{array}$ & $20(32.8 \%$ & $19(31.1 \%$ & $18(29.5 \%$ & 0.365 \\
\hline \multicolumn{7}{|c|}{ Educational Background } \\
\hline $\begin{array}{l}\text { Vocational } \\
\text { college }\end{array}$ & & $6(9.8 \%)$ & $3(4.9 \%)$ & $1(1.6 \%)$ & $2(3.2 \%)$ & \\
\hline $\begin{array}{l}\text { Junior } \\
\text { college }\end{array}$ & & $5(8.2 \%)$ & $1(1.6 \%)$ & $3(4.9 \%)$ & $1(1.6 \%)$ & \\
\hline
\end{tabular}


Citation: Nishimura A (2016) Effects of Different Methods for Achieving Situational Awareness on Cerebral Blood Flow in Nurses in an Observational Setting. Gen Med (Los Angeles) 4: 280. doi:10.4172/2327-5146.1000280

Page 4 of 7

\begin{tabular}{|l|l|l|l|l|l|l|}
\hline College & $\begin{array}{l}46(75.4 \% \\
)\end{array}$ & $17(27.9 \%$ & $15(24.6 \%$ & $14(23.0 \%$ & \\
\hline & & $4(6.6 \%)$ & $0(0 \%)$ & $1(1.6 \%)$ & $3(4.9 \%)$ & 0.176 \\
\hline $\begin{array}{l}\text { Graduate } \\
\text { School }\end{array}$ & $5.1(\mathrm{Q} 2.5)$ & $5.1(\mathrm{Q} 2.8)$ & $4.6(\mathrm{Q} 2.6)$ & $5.1(\mathrm{Q} 2.3)$ & 0.705 \\
\hline $\begin{array}{l}\text { Years of } \\
\text { experienc } \\
\mathrm{e}\end{array}$ & $5.0(\mathrm{Q} 2.5)$ & $5.1(\mathrm{Q} 3.1)$ & $4.1(\mathrm{Q} 4.5)$ & $5.1(\mathrm{Q} 1.5)$ & 0.809 \\
\hline $\begin{array}{l}\text { Experienc } \\
\mathrm{e} \text { in the } \\
\text { ward }\end{array}$ & & & & \\
\hline \multicolumn{7}{|l|}{ Fisher's exact test or Kruskal- Wallis test } \\
\hline
\end{tabular}

Table 1: Attributes of participants

\begin{tabular}{|l|l|l|l|l|l|}
\hline & All & Writing & Oral & Implicit & \\
\hline & $\mathbf{n = 1 6}$ & $\mathbf{n = 2 1}$ & $\mathbf{n = 2 0}$ & $\mathbf{n}=\mathbf{2 0}$ & $\begin{array}{l}\mathbf{p} \\
\text { value }\end{array}$ \\
\hline $\begin{array}{l}\text { Intuitive } \\
\text { judgement }\end{array}$ & $62.0(\mathrm{Q} 9.5)$ & $64.0(\mathrm{Q} 8.8)$ & $62.0(\mathrm{Q} 7.2)$ & $61.5(\mathrm{Q} 7.2)$ & 0.713 \\
\hline $\begin{array}{l}\text { Local } \\
\text { Judgement }\end{array}$ & $70.0(\mathrm{Q} 7.8)$ & $73.0(\mathrm{Q} 11.8)$ & $71.0(\mathrm{Q} 7.8)$ & $66.5(\mathrm{Q} 10.3)$ & 0.106 \\
\hline $\begin{array}{l}\text { Mental } \\
\text { Summarization }\end{array}$ & $51.0(\mathrm{Q} 12.8)$ & $52.0(\mathrm{Q} 12.5)$ & $50.5(\mathrm{Q} 13.5)$ & $51.0(\mathrm{Q} 14.4)$ & 0.746 \\
\hline $\begin{array}{l}\text { Verbal } \\
\text { Description }\end{array}$ & $45.0(\mathrm{Q} 12)$ & $45.0(\mathrm{Q} 10.5)$ & $51.0(\mathrm{Q} 15.8)$ & $41.5(\mathrm{Q} 12.9)$ & 0.538 \\
\hline \begin{tabular}{l} 
Writing \\
\hline $\begin{array}{l}\text { Touch Panel } \\
\text { Usage }\end{array}$
\end{tabular} & $55.0(\mathrm{Q} 13.3)$ & $57.0(\mathrm{Q} 12.0)$ & $53.0(\mathrm{Q} 13.0)$ & $43.0(\mathrm{Q} 11.4)$ & 0.303 \\
\hline $\begin{array}{l}\text { Drowsiness on } \\
\text { the day of } \\
\text { experiment }\end{array}$ & $44.0(\mathrm{Q} 26.3)$ & $51.0(\mathrm{Q} 16.5)$ & $54.5(\mathrm{Q} 14.2)$ & $56.5(\mathrm{Q} 15.8)$ & 0.488 \\
\hline $\begin{array}{l}\text { Fatigue on the } \\
\text { day } \\
\text { experiment }\end{array}$ & $49.0(\mathrm{Q} 24.0)$ & $48.0(\mathrm{Q} 17.8)$ & $55.0(\mathrm{Q} 27.9)$ & 0.135 \\
\hline $\begin{array}{l}\text { Kruskal- Wallis Test } \\
\text { Kras }\end{array}$ & $34.0(\mathrm{Q} 28.3)$ & $48.0(\mathrm{Q} 19.2)$ & $57.0(\mathrm{Q} 25.2)$ & 0.659 \\
\hline
\end{tabular}

Table 2: Attributes of participants (VAS)

\section{Changes in cerebral blood flow (Figure 2) and VAS (Table 3) during the task}

During the task before situational awareness, total $\mathrm{Hb}$ values on the left (left total $\mathrm{Hb}$ values) in the writing, oral, and implicit groups were 0.51 (Q 0.22), 0.59 ( $\mathrm{Q} 0.47$ ), and 0.82 (Q 0.56), respectively; total $\mathrm{Hb}$ values on the right (right total $\mathrm{Hb}$ values) were $0.73(\mathrm{Q} 0.44), 0.62(\mathrm{Q}$ 0.58 ), and 0.78 ( $Q$ 0.57), respectively. There were no significant differences in this respect on both sides $(\mathrm{p}>0.05)$. In contrast, during the task after situational awareness, left total $\mathrm{Hb}$ values in the writing, oral, and implicit groups were $0.85(\mathrm{Q} 0.85), 1.06(\mathrm{Q} 0.78)$, and $0.55(\mathrm{Q}$ 0.49 ), respectively, and the right total $\mathrm{Hb}$ values were $1.13(\mathrm{Q} 0.66)$, 1.11 (Q 0.85), and 0.68 (Q 0.50), respectively. There was a significant difference between the oral group and the implicit group $(\mathrm{p}=0.019)$; the right total $\mathrm{Hb}$ value in the oral group was the highest among the three (Figure 2).

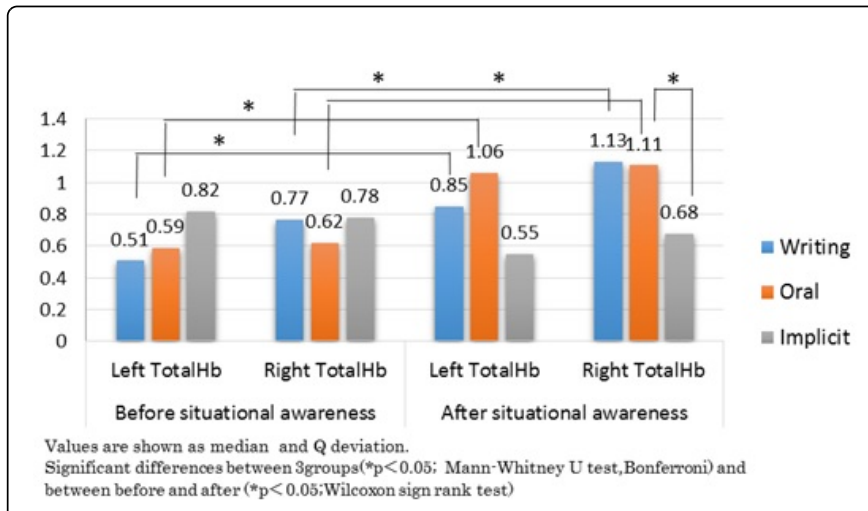

Figure 2: Changes in cerebral blood flow before and after situation awareness

On comparing the values for the pre- and post-situational awareness tasks, significant differences were observed for the left total $\mathrm{Hb}$ $(\mathrm{p}=0.000)$ and right total $\mathrm{Hb}(\mathrm{p}=0.004)$ values in the writing group and for the left total $\mathrm{Hb}(\mathrm{p}=0.010)$ and right total $\mathrm{Hb}(\mathrm{p}=0.005)$ values in the oral group; post-situational awareness values were better in all cases; however, the difference was not statistically significant in the implicit group (Table 3).

\begin{tabular}{|c|c|c|c|c|c|c|c|c|}
\hline & \multicolumn{3}{|c|}{ Before Situational awareness } & & \multicolumn{3}{|c|}{ After Situational awareness } & \\
\hline & Writing & Oral & Implicit & & Writing & Oral & Implicit & \\
\hline & $\mathrm{n}=21$ & $\mathrm{n}=20$ & $\mathrm{n}=20$ & $p$ value & $\mathrm{n}=21$ & $\mathrm{n}=20$ & $\mathrm{n}=20$ & $p$ value \\
\hline $\begin{array}{l}\text { Consciously } \\
\text { observed }\end{array}$ & $\begin{array}{l}69.0 \\
(Q 9.3)\end{array}$ & $\begin{array}{l}70.0 \\
(Q 11.0)\end{array}$ & $\begin{array}{l}74.0 \\
\text { (Q93.5) }\end{array}$ & 0.91 & $\begin{array}{l}74.0 \\
(Q 9.0)\end{array}$ & $\begin{array}{l}77.0 \\
(Q 7.5)\end{array}$ & $\begin{array}{l}73.5 \\
(\mathrm{Q} 8.0)\end{array}$ & 0.71 \\
\hline $\begin{array}{l}\text { Repeatedly } \\
\text { Observed }\end{array}$ & $\begin{array}{l}69.0 \\
(Q 9.2)\end{array}$ & $\begin{array}{l}70.5 \\
(Q 9.2)\end{array}$ & $\begin{array}{l}71.0 \\
(Q 6.5)\end{array}$ & 0.93 & $\begin{array}{l}80.0 \\
(Q 11.5)\end{array}$ & $\begin{array}{l}69.50 \\
(Q 15.0)\end{array}$ & $\begin{array}{l}70.5 \\
(\mathrm{Q} 12.5)\end{array}$ & 0.05 \\
\hline $\begin{array}{l}\text { Observed while } \\
\text { thinking } \\
\text { about a reason }\end{array}$ & $\begin{array}{l}76.0 \\
(\mathrm{Q} 6.8)\end{array}$ & $\begin{array}{l}75.0 \\
(Q 8.6)\end{array}$ & $\begin{array}{l}76.0 \\
(\mathrm{Q} 13.4)\end{array}$ & 0.74 & $\begin{array}{l}77.0 \\
\text { (Q15.5) }\end{array}$ & $\begin{array}{l}81.0 \\
(Q 9.0)\end{array}$ & $\begin{array}{l}81.0 \\
(Q 9.0)\end{array}$ & 0.12 \\
\hline
\end{tabular}


Table 3: Change in subject evaluation before and after situational awareness( VAS)

When VAS was used for scoring after the situational awareness task, there were no significant differences in intergroup comparisons before or after situational awareness. In the comparisons between before versus after situational awareness, the VAS for "repeatedly observed" in the writing group significantly increased after situational awareness $(\mathrm{p}=0.02)$.

\section{Intergroup comparison of cerebral blood flow at the situational awareness opportunity (Figure 3)}

With respect to changes in cerebral blood flow during situational awareness, left total $\mathrm{Hb}$ values in the writing, oral, and implicit groups were 1.07 (Q 0.63), 1.41 (Q 0.59), and 0.18 (Q 0.37), respectively; a significant difference between the writing group and the oral group was observed in this respect $(\mathrm{p}=0.00)$. Right total $\mathrm{Hb}$ values in the writing, oral, and implicit groups were 1.46 (Q 0.51), 2.14 (Q 1.01), and 0.53 (Q 0.42); significant differences in this respect were observed between the writing group and the implicit group and between the oral group and the implicit group $(\mathrm{p}=0.00, \mathrm{p}=0.0)$ (Figure 3 ).

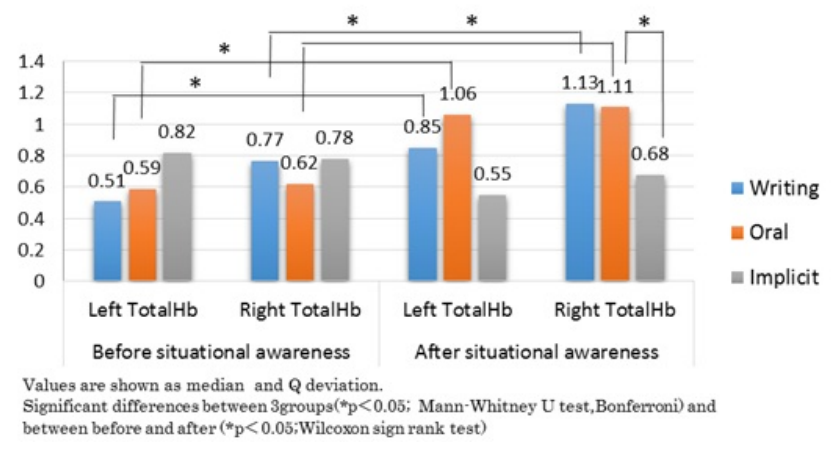

Figure 3: Intergroup comparison of cerebral blood flow at situational awareness opportunity

\section{Discussion}

\section{Effects of creating opportunities to practice situational awareness on cerebral blood flow}

The effects that providing opportunities to practice situational awareness had on cerebral blood flow were identified when we compared pre- and post-situational awareness values. These were as follows: (1) left and right total $\mathrm{Hb}$ values increased after situational awareness in the writing and oral groups; (2) right total $\mathrm{Hb}$ values in the pre-situational awareness task in the oral group were high; and (3) left and right total $\mathrm{Hb}$ values during situational awareness were high in the oral group.

Because total $\mathrm{Hb}$ in the writing and oral groups significantly increased during observation while performing the task after situational awareness, we thought that the opportunity to use the writing or oral method to achieve situational awareness enhanced the brain activity and approximated the changes associated with more intensive thinking. On the other hand, no significant differences were observed between pre- and post-situational awareness total $\mathrm{Hb}$ values in the implicit group. We therefore believe that despite the fact that opportunities to practice situational awareness were provided, there were no effects on the brain activity when an implicit method was used. As the left and right total $\mathrm{Hb}$ values in the implicit group were both lower than those in the oral group, similar to the findings for cerebral blood flow during situational awareness, we now know that brain activity during these times is suppressed.

The prefrontal cortex, which was used as a measurement index during the present study, performs activities associated with executive function. These include working memory, which is a mechanism for short-term retention and processing of information and performing goal-oriented activities [12]. Executive function is the ability to perform voluntary, purposeful actions; it includes setting intentions and objectives, developing plans, making purposeful goal-oriented actions, and acting effectively [12]. The task in this study was to observe a still image of a hospital room as a nurse. When nurses perform observation, executive function is important for helping them understand the current situation and predict the future based on past experiences and knowledge. Therefore, executive function of the prefrontal cortex is important for a nurse. The enhanced prefrontal cortex activity when practicing situational awareness in the writing and oral groups and the subsequent observations most likely reflect the effect of thinking and a conscious effort to observe more attentively, thus leading to active situational awareness.

Broca's area forms the largest portion of the outer left prefrontal cortex. This area is involved in language activities such as speech, verbal thinking, and grammar [14]. The left prefrontal cortex is activated during a verbal working memory task [15]. The right hemisphere has also been verified to be predominant during visual spatial perception by means of event-related potential and other means $[16,17]$. The right dorsolateral portion in particular has been shown to be involved in switching visuospatial attention and controlling cognitive processes for the processing of the central execution system [18].

The results of this study suggest that effects of situational awareness on the subsequent observation were not limited to facilitation of verbal understanding as an execution function, but also extended to visual perception as cerebral blood flow in the left and right prefrontal areas increased during the induced situational awareness by writing and oral means, which are expressed as language. This is also supported by significantly increased VAS scores for "repeatedly observed" in the writing group, and there seems to be a visual appeal that urges participants to repeatedly watch the hospital room environment in the task picture.

These findings suggest that situational awareness was achieved in the writing and oral groups; this occurred during situational awareness as well as during observation after situational awareness. These factors acted on natural decision making to result in enhanced brain activity during observation after situational awareness. 
Page 6 of 7

\section{Future utilization of the opportunity to perform situational awareness}

From the standpoint of brain activity and current understanding, it has been reported that habitual visual image stimuli, such as those encountered during games, are not transmitted as information to the prefrontal cortex [19]. In other words, one starts responding to stimuli reflexively without questioning or thinking, which retains the ambiguous understanding as is. The same phenomenon may be applicable to nurses; making judgments and actions based on previous experiences without conscious reasoning in practical settings is likely to lead to inappropriate natural decisions. This may in turn increase the probability of errors. For nurses to protect patient safety and to practice nursing skills supported by scientific evidence using the latest knowledge and technologies, it is important that nurses as practitioners learn to develop situational awareness in the context of the action, reflect on implicit knowledge during the action, expressly question this knowledge, and reflect and understand it in order to implement future actions [20,21].

The purpose of inducing situational awareness by using writing and oral methods in the present study was to formalize the thinking of situational awareness during observation. This method facilitated the action of the prefrontal cortex not only during situational awareness but also in the subsequent observation. In other words, after situational awareness, the observation was likely to be accompanied by thinking, rather than a mere reflexive response to the stimulus of the still image. This indicates that the situational awareness not only affects that scene but also the subsequent observations. Conversely, the implicit method did not act on the prefrontal cortex and made no difference to the subsequent observation.

These findings suggest that opportunities to formalize the thinking of situational awareness through writing and oral means may be utilized in nursing practice in the future.

\section{Study limitations and future tasks}

No significant differences in cerebral blood flow were found between the writing and oral groups in this study. In addition, we did not study the quality or quantity of verbalized content in response to the situational awareness stimulus. Writing and oral methods are identical in terms of formalizing thought processes but are differentially processed to achieve it. Therefore, effects of different situational awareness methods other than those on cerebral blood flow and the analysis of formalized content remain to be studied in the future.

\section{Conclusion}

The effects that providing opportunities to practice situational awareness had on cerebral blood flow were identified when we compared pre- and post-situational awareness values. These were as follows: (1) left and right total $\mathrm{Hb}$ values increased after situational awareness in the writing and oral groups; (2) right total $\mathrm{Hb}$ values in the pre-situational awareness task in the oral group were high; and (3) left and right total $\mathrm{Hb}$ values during situational awareness were high in the oral group. The use of writing and oral methods by nurses for situational awareness was found to promote the brain activity not only during the process of situational awareness but also during the observations after situational awareness.

\section{Competing interests}

The authors declare that they have no competing interests.

\section{Acknowledgment}

I would like to express my deepest gratitude to all participants who cooperated in the present study. This study was supported by Grant-inAid for Young Researchers from Japan Society of Private Colleges and Universities of Nursing and by placement of research assistants from Tokyo Medical University by Program to Supporting Research Activities of Female Researchers as a part of Program for Human Resource Development Program in Science and Technology by the Ministry of Education, Culture, Sports, Science and Technology.

\section{References}

1. Koch SH, Weir C, Haar M, Staggers N, Agutter J, et al. (2012) Intensive care unit nurses' information needs and recommendations for integrated displays to improve nurses' situation awareness. J Am Med Inform Assoc 19: 583-590.

2. Wright MC, Taekman JM, Endsley MR (2004) Objective measures of situation awareness in a simulated medical environment. Qual Saf Health Care 13: i65-i71.

3. McKenna L, Missen K, Cooper S, Bogossian F, Bucknall T, et al. (2014) Situation awareness in undergraduate nursing students managing simulated patient deterioration. Nurse Educ Today 34: e27-e31.

4. Stubbings L, Chaboyer W, McMurray A (2012) Nurse's use of situation awareness in decision-making: an integrative review. J Adv Nurs 68: 1443-1453.

5. Taylor J, Sims J, Haines TP (2014) The emergent relevance of care staff decision-making and situation awareness to mobility care in nursing homes: an ethnographic study. J Adv Nurs 70: 2767-2778.

6. Ishizaka M, Takeda K, Shimoi T, Maruyama H (2012) Relationship between Intensity of Toe Motion and Cerebral Activation : a NearInfrared Spectroscopy Study. The Society of Physical Therapy Science 27: 165-170.

7. Goswami U (2006) Neuroscience and education: from research to practice? Nat Rev Neurosci 7: 406-411.

8. Strangman G, Boas DA, Sutton JP (2002) Non-invasive neuroimaging using near-infrared light. Biol Psychiatry 52: 679-693.

9. Boas DA, Elwell CE, Ferrari M, Taga G (2014) Twenty years of functional near-infrared spectroscopy: introduction for the special issue. Neuroimage 85: 1-5.

10. Kuroda Y, Okamoto N, Maesako T (2015) Possibility of Research in Educational Neuroscience by Measurement of Brain Activity Using Near Infra-red Spectroscopy. The Journal of Japan Society for Laser Surgery and Medicine 36: 176-184.

11. Ohsugi H, Ohgi S, Kodama T (2014) Physical activity affects cognitive function in view of prefrontal cortex activation. Journal of Allied Health Sciences 5: 69-77.

12. Lezak MD (1995) Neuropsychological assessment. 3rd ed.Oxford University Press, NY.

13. Baddely A (1986) Working memory. Oxford University Press, Oxford.

14. Bear MF, Connors BW, Paradiso MA (2007) Neuroscience: exploring the brain. 3rd ed.Lippincott Williams \& Wilkins, Philadelphia, Baltimore, Tokyo.

15. Leon-Dominguez U, Martin-Rodriguez JF, Leon-Carrion J (2015 ) Executive n-back tasks for the neuropsychological assessment of working memory. Behav Brain Res 292:167-173.

16. Daniel TA, Katz JS, Robinson JL ( 2016) Delayed match-to-sample in working memory: A BrainMap meta-analysis. Biol Psychol 120:10-20.

17. Numata K, Nakajima Y, Shimizu S (1998) Right Hemisphere Dominance for Figure Recognition: A Study of Event Related Potentials to Lateralized 
Citation: Nishimura A (2016) Effects of Different Methods for Achieving Situational Awareness on Cerebral Blood Flow in Nurses in an Observational Setting. Gen Med (Los Angeles) 4: 280. doi:10.4172/2327-5146.1000280

Page 7 of 7

Projection. Journal of the Japanese Physical Therapy Association 12 45-52.

18. Kondo H, Osaka N, Osaka M (2004) Cooperation of the anterior cingulate cortex and dorsolateral prefrontal cortex for attention shifting. Neuroimage 23: 670-679.

19. Beritashvili IS (1972) The role of prefrontal lobes in psychonervous memory in vertebrates. Acta Neurobiol Exp (Wars) 32: 657-672.
20. Schon DA (1983) The Reflective Practitioner : How professionals Think in Action. Basic Books, New York.

21. Burns S, Bulman C (2000) Reflective practice in nursing: the growth of the professional practitioner. Blackwell Science, Oxford. 